\title{
SER/BER expression for M-QAM OFDM systems with imperfect channel estimation and I/Q imbalance
}

\author{
Udesh Oruthota* and Olav Tirkkonen
}

\begin{abstract}
We investigate the joint effect of channel estimation and frequency flat transmitter and receiver I/Q imbalance on an Orthogonal Frequency Division Multiplex (OFDM) system. We assume independent fading with identically and independently distributed (i.i.d) mirror carrier channel coefficients. Closed form expressions for symbol error rate and bit error rate of M-QAM modulation are derived. We consider join and separate channel estimation, as well as joint and separate equalization of the mirror carriers. Performance is evaluated by treating the I/Q interference as a non-Gaussian random variable. The results show that a Gaussian approximation of I/Q interference is very good, especially for low order modulations.
\end{abstract}

Keywords: I/Q imbalance, OFDM, Rayleigh fading channel, Imperfect channel estimation, Symbol error rate, Bit error rate

\section{Introduction}

Orthogonal frequency division multiplexing (OFDM) based transceivers have been selected for several wireless standards such as IEEE 802.11, LTE and WiMax, and are the likely selection for 4th generation systems fulfilling the International Mobile Telephony (IMT)Advanced requirements [1]. Low-cost implementation of transceiver devices is challenging in view of impairments associated with imperfect analog components. Imperfections due to power amplifier (PA) non-linearity, frequency offset, phase noise, timing jitter, quantization noise and I/Q imbalance distort the baseband signal [2]. The most challenging IMT-Advanced requirements are for local area scenarios [1]. In local area, transmission powers are comparatively small, which alleviates problems with PA non-linearity. In OFDM systems, carrier frequency offset, phase noise and timing jitter cause inter-carrier interference (ICI) which is strongest between neighboring subcarriers, and their relative strength is related to the subcarrier bandwidth. Their effect may thus be reduced by selecting a suitable OFDM parametrization. With increasing computational capabilities, the number

\footnotetext{
*Correspondence: Udesh.Oruthota@aalto.fi
}

Department of Communications \& Networking, Aalto University School of

Science and Technology, P.O. Box 13000, Fl-02150, Aalto, Finland of bits in analog-to-digital conversion may be increased, reducing quantization noise. Thus the only impairment left without a straight forward engineering cure is I/Q imbalance.

Different approaches for digital compensation of the I/Q imbalance have been proposed [3-5]. The goal of compensation is to provide an improved image rejection, which by nature depends on the accuracy of the digital estimation and compensation approaches used.

Knowledge about the quantitative relationship between transceiver parameters such as the image rejection ratio (IRR) and system parameters such as the symbol error probability is essential for the design and dimensioning of communications systems. In practice, channel estimation cannot be done perfectly in a fading environment.

Several solutions have been proposed in the literature to estimate the I/Q imbalance parameters, and to compensate in analog and digital domains [4-11]. Blind estimation and compensation techniques [5], interference cancelation based [6], pilot aided and preamble based techniques have been discussed to estimate the channel and the I/Q imbalance parameters [8-10]. Joint estimation of channel and the $\mathrm{I} / \mathrm{Q}$ parameters can be found in [11].

\section{Springer}

(C) 2012 Oruthota and Tirkkonen; licensee Springer. This is an Open Access article distributed under the terms of the Creative Commons Attribution License (http://creativecommons.org/licenses/by/2.0), which permits unrestricted use, distribution, and reproduction in any medium, provided the original work is properly cited. 
The I/Q imbalance compensation for different fading environments and modulation orders, have been investigated in [3]. Performance degradation due to I/Q imbalance under perfect channel knowledge is evaluated in [12]. It is a challenging task to implement computationally efficient estimation techniques while minimizing the estimation error with the impact of I/Q imbalance.

Krondorf and Fettwis $[13,14]$ numerically evaluate the bit error rate performance of a coded and uncoded OFDM systems with all impairments including carrier frequency offset (CFO), I/Q imbalance, frequency selectivity and channel estimation errors on direct conversion $\mathrm{ZF}$ receiver with Least Square (LS) channel estimation. The inter-carrier interference (ICI) due to CFO and I/Q imbalance on effective transmitted symbol is assumed to be Gaussian distributed.

In this article, we concentrate on digital compensation of the I/Q imbalance. The goal is to understand system performance of three different estimation and equalization schemes which are commonly used in communication systems. The baseline solution is separate channel estimation and separate equalization of mirror subcarriers. This is contrasted with joint channel estimation with separate or joint equalization. We present closed form expressions for both the symbol error rate (SER) and the bit error rate (BER) with Gray coded bit mapping in independently Rayleigh fading channels under the joint effects of least squares channel estimation and frequency flat transmitter and receiver I/Q imbalance. Comparing to the Gaussian BER approximation results of [13], we observe that the Gaussian approximation works well, especially for small constellation sizes.

The remainder of this article is organized as follows. Section 'System model' describes the system model under the effect of I/Q imbalance and imperfect channel estimation. In Section 'Signal models with channel estimation errors', we evaluate the error displacement vector for three different channel estimation and equalization schemes related to joint/separate treatment of the mirror subcarriers. In Section 'M-QAM symbol error rate', we calculate the SER based on displacement error vector analysis. It is further simplified using the power distribution pattern of the desired and mirror signals. Then, the previous result is extended towards BER in Section 'Bit error rate for M-QAM symbol transmission' with a closed form solution for square M-QAM modulations. Analytical limits for perfect channel knowledge are addressed in Section 'Analytical limits'. Ultimately, simulation results and conclusions are made in Sections 'Simulation results' and 'Conclusion', respectively.

Notation. $\mathrm{E}(\cdot), Q(\cdot), \operatorname{erfc}(\cdot)$, and $\mathrm{P}(\mathrm{E})$ symbolize the expectation operator, the Gaussian Q-function, complementary error function and the error probability, respectively. The superscripts $*$ stands for complex conjugate and $\operatorname{det}(\cdot)$ and $|\cdot|$ refer the matrix determinant and absolute value in that order.

\section{System model}

We consider the effect of transmitter and receiver I/Qimbalance on channel estimation in broadband multicarrier communication with direct conversion transceivers.

\section{I/Q imbalance model}

In the down-conversion of an ideal direct conversion receiver, the incoming signals in the I (in-phase) and Q (quadrature) branches are perfectly match with gains and orthogonal in phase. However, real receivers introduce gain mismatch and non-orthogonality in phase of I and $\mathrm{Q}$ branches due to imperfect electronic implementation. These imperfections at the demodulator can be seen as a receiver I/Q imbalance in baseband domain [3]. Similarly, the phase and gain mismatch of the modulator introduce the transmitter I/Q imbalance.

The transmitter I/Q imbalance can be modeled as $s(t)=$ $G_{1} x(t)+G_{2}^{*} x^{*}(t)$ where $s(t)$ is the output at the modulator after I/Q corruption and $x(t)$ is the ideal baseband equivalent transmitted signal under perfect matching. In a same fashion demodulator output after receiver I/Q imbalance is $y(t)=K_{1} r(t)+K_{2} r^{*}(t)$ where $r(t)$ is the input signal at the receiver front end which is corrupted by the channel $h(t) \sim \mathcal{C N}\left(0, \sigma_{h}^{2}\right)$ and noise $n(t) \sim \mathcal{C N}\left(0, N_{0}\right)$. Both transmitter and receiver I/Q imbalance coefficients $G_{1}=\left(1+g_{T} e^{j \phi_{T}}\right) / 2, G_{2}=\left(1-g_{T} e^{-j \phi_{T}}\right) / 2$ and $K_{1}=$ $\left(1+g_{R} e^{-j \phi_{R}}\right) / 2, K_{2}=\left(1-g_{R} e^{j \phi_{R}}\right) / 2$ are the complex coefficients which can be determined by the total effective amplitude and phase imbalances of the transceivers, $g$ and $\phi$, respectively. Notice that the above impairment model assumes frequency-flat I/Q imbalance within the signal band which is a rather typical assumption in this context.

The above I/Q imbalance model corresponds to the following transformations of the effective baseband equivalent signals at the receiver output

$$
\begin{aligned}
y(t)= & K_{1}(s(t) \otimes h(t))+K_{2}(s(t) \otimes h(t))^{*} \\
& +K_{1} n(t)+K_{2} n(t)^{*} .
\end{aligned}
$$

In the equivalent frequency domain signal, there is ICI between mirror carrier pairs which are located symmetrically around the center subcarrier [10] (see Figure 1);

$$
y_{+}=h_{++} x_{+}+h_{+-} x_{-}^{*}+K_{1} n_{+}+K_{2} n_{-}^{*},
$$

where $h_{++}=K_{1} h_{+} G_{1}+K_{2} h_{-}^{*} G_{2}, h_{+-}=K_{1} h_{+} G_{2}^{*}+$ $K_{2} h_{-}^{*} G_{1}^{*}$ are the I/Q modulated frequency domain channel coefficients and $h_{+}$and $h_{-}$are the channel coefficients on mirror carrier pair. The received signal on subcarrier is $y_{+}$and the corresponding transmitted symbols on mirror carriers are $x_{+}$and $x_{-}$. Similarly noise samples can be defined. 


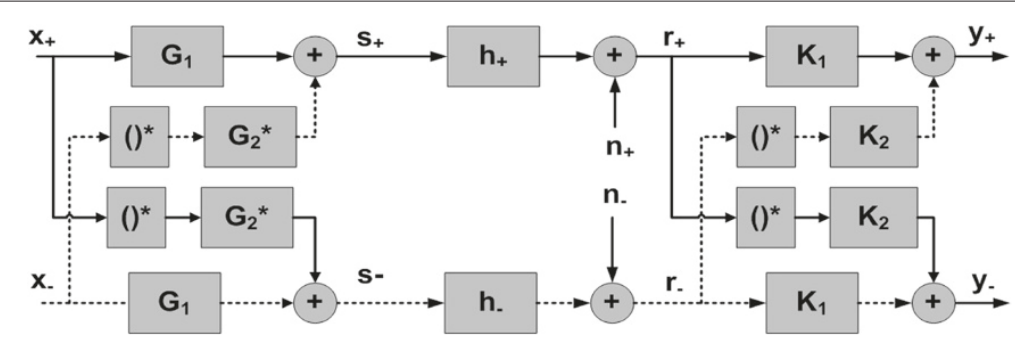

Figure 1 Block diagram representation of an OFDM system with transmitter and receiver I/Q imbalances. The subcarrier and mirror carrier channels are $h_{+}$and $h_{-}$and the transmitter and receiver I/Q imbalance coefficients are $G_{1}, G_{2}$ and $K_{1}, K_{2}$, respectively.

The mirror-frequency attenuation is described by the image rejection ratio (IRR) of the receiver RF front-end, $\left|\frac{K_{1}}{K_{2}}\right|^{2}$, which is typically in the order of $25-40 \mathrm{~dB}$ [15] in practical receivers. Below, we shall frequently use the inverse IRR, the image leakage ratio, $K=\left|\frac{K_{2}}{K_{1}}\right|^{2}$ in the analysis.

\section{Channel estimation error model}

In general, the estimation error for a channel $c$ is given by $e=\hat{c}-c$, where $\hat{c}$ indicates the channel estimate. Only for minimum mean square error-channel estimation, the error term $e$ and the estimated channel $\hat{c}$ are uncorrelated; $\mathrm{E}\left\{e^{*} \hat{c}\right\}=0$ [16]. A versatile channel estimation model in [17] describes the correlated channel estimation error for an arbitrary linear channel estimators. The model decouples the correlation between $\hat{c}$ and $e$ by introducing a new uncorrelated random variable $(\mathrm{RV}) z$,

$$
c=a \hat{c}+z
$$

where $a=\rho \frac{\sigma_{c}}{\hat{\sigma}_{c}}$ is the biasing factor and $\sigma_{c}^{2}$ and $\hat{\sigma}_{c}^{2}$ are the variances of the channel and its estimate. The normalized correlation coefficient is $\rho=\mathrm{E}\left\{\hat{c}^{*} c\right\} / \sigma_{c} \hat{\sigma}_{c}$. The uncorrelated estimation error $z$ can be obtained from a complex Gaussian process $\mathcal{C N}\left(0, \sigma_{z}^{2}\right)$, where $\sigma_{z}^{2}=\left(1-|\rho|^{2}\right) \sigma_{c}^{2}$.

LS channel estimation is a subset of the above error model and error $e$ in general is the zero mean with a certain variance due to effective noise and it is independent of $c$ over a slow fading channels [16]. Using (3), we can decouple the LS estimation error to uncorrelated $z$ and a biasing factor. Then $\rho^{2}=\frac{\sigma_{c}^{2}}{\sigma_{c}^{2}+\sigma_{e}^{2}}$, where $\sigma_{e}^{2}$ is the error variance. Hence, the biasing factor becomes $a=\frac{\sigma_{c}^{2}}{\sigma_{c}^{2}+\sigma_{e}^{2}}=$ $\rho^{2}$. For LS estimation, the biasing factor is directly given by the correlation coefficient. If error $e$ and channel $c$ are correlated to each other then the biasing factor becomes $a=\mathrm{E}\left\{\hat{c}^{*} c\right\} / \hat{\sigma}_{c}^{2}$ and same channel estimation error model can be still valid.

\section{Signal models with channel estimation errors}

This article considers different combinations of I/Qimbalance aware channel estimation and equalization. Both estimation and equalization can be performed separately or jointly for the mirror subcarrier pair. Three combinations make sense: (i) Baseline solution with separate channel estimation and separate equalization. (ii) Joint channel estimation but separate equalization. (iii) Joint channel estimation and joint equalization. These can be easily described under the channel estimation error model (3), and a similar error displacement vector model.

Frequency selective channels with independently fading mirror carrier pairs are considered. This is a realistic assumption in broadband communications where the channel coherence bandwidth is typically smaller than the frequency separation between mirror carrier pair. We consider LS estimation throughout.

The pair of I/Q modulated channel coefficients $h_{++}$ and $h_{+-}$at each subcarrier index can be estimated either separately or jointly, using a known sequence of pilots. The I/Q corrupted signal models with channel estimation errors can be constructed based on (2) for the three cases considered.

\section{Separate channel estimation with separate equalization}

A known BPSK pilot sequence is inserted in to the OFDM symbol to estimate the I/Q modulated channel $h_{++}=$ $K_{1} h_{+} G_{1}+K_{2} h_{-}^{*} G_{2}$ on the $k$ th subcarrier, assuming the channel is time invariant under several OFDM symbols. The receiver estimates the channel without awareness of the I/Q imbalance, using a LS estimator. Using (2), the estimated channel becomes

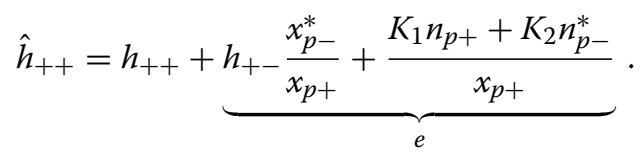

For known pilots $x_{p+}$ and $x_{p-}$ on the mirror carrier pair, the estimation error becomes a complex Gaussian RV with zero mean and the variance $\sigma_{e}^{2}=\left(\left|K_{1} G_{2}\right|^{2}+\right.$ $\left.\left|K_{2} G_{1}\right|^{2}\right) \sigma_{h}^{2}+N_{p}\left(\left|K_{1}\right|^{2}+\left|K_{2}\right|^{2}\right)$. The biasing factor then 
becomes $a=\frac{\left|\sigma_{h++}^{2}+\eta\right|}{\sigma_{h++}^{2}+\sigma_{e}^{2}+2 \Re\{\eta\}}$ where $\eta=\left(\left|K_{1}\right|^{2}+\left|K_{2}\right|^{2}\right)$ $\sigma_{h}^{2} G_{1} G_{2}$ is the correlation $\mathrm{E}\left\{e_{+}^{*} h_{++}\right\}$with same pilot transmission on mirror carrier pair. The noise power spectral density of the pilot transmission is given by $N_{p}$ and $\sigma_{h}^{2}$ is the variance of the physical channel $h_{+}$(not of the channel to be estimated, $h_{++}$).

After single tap equalization, the estimate of the data symbol on the subcarrier of interest is

$$
\hat{x}_{+}=x_{+}+\frac{z_{++} x_{+}+h_{+-} x_{-}^{*}+K_{1} n_{+}+K_{2} n_{-}^{*}}{a \hat{h}_{++}} .
$$

The error displacement vector, characterizing the symbol estimate error becomes

$$
\Delta=\hat{x}_{+}-x_{+}=\frac{z_{++} x_{+}+h_{+-} x_{-}^{*}+K_{1} n_{+}+K_{2} n_{-}^{*}}{a \hat{h}_{++}},
$$

where the estimation error $z_{++}$is independent from $\hat{h}_{++}$ by definition and $n_{+}, n_{-}$are also independent processes. However, there is a correlation exist between $h_{+-}$and $\hat{h}_{++}$. Hence, $\Delta$ can be modeled as a fraction of two correlated complex Gaussian RVs when conditioned on a given set of transmitted symbols on mirror subcarrier pair.

\section{Joint channel estimation and separate equalization}

Here, we consider a receiver where there is a simple pilot structure to mitigate the effect of the I/Q imbalance on the channel estimation, but where I/Q compensation is not considered in equalization. This case was analyzed in [18]. We formulate the analysis in terms of estimating the full $\mathbf{h}$ until explicit equalization is performed. To remove the effect of I/Q imbalance from channel estimation, pilot transmission from two consecutive OFDM symbols is considered and the pilot symbols transmitted on these subcarriers are

$$
\mathbf{X}_{p}=\left[\begin{array}{ll}
x_{1+} & x_{1-}^{*} \\
x_{2+} & x_{2-}^{*}
\end{array}\right]
$$

where $x_{j}, j=1,2$ are the pilot data symbols in two consecutive OFDM symbols. These are selected so that $\mathbf{X}_{p}$ becomes non-singular for each mirror subcarrier pair, i.e., det $\left(\mathbf{X}_{p}\right)=\left|x_{1+} x_{2-}^{*}-x_{1-}^{*} x_{2+}\right| \neq 0$. A simple selection maintaining the orthogonality between subcarriers is based on a Walsh-Hadamard matrix [10]; $x_{j+}=1, x_{1-}=$ -1 , and $x_{2-}=1$. The estimated channel coefficients $\hat{\mathbf{h}}$ are functions of these pilot data symbols. The LS estimate of the channel is $\hat{\mathbf{h}}=\mathbf{h}+\mathbf{X}_{p}^{-1} \overline{\mathbf{n}}$. Here the elements of the I/Q corrupted noise vector $\overline{\mathbf{n}}$ are $\bar{n}_{i}=K_{1} n_{i+}+K_{2} n_{i-}^{*}$. In terms of the channel estimation error, the received signal is

$$
y=\left(a \hat{h}_{++}+z_{++}\right) x_{+}+h_{+-} x_{-}^{*}+K_{1} n_{+}+K_{2} n_{-}^{*}
$$

Single-tap zero-forcing equalization against $a \hat{h}_{++}$, assuming channel statistics are known to the receiver, gives the error displacement vector

$$
\Delta=\frac{z_{++} x_{+}+h_{+-} x_{-}^{*}+K_{1} n_{+}+K_{2} n_{-}^{*}}{a \hat{h}_{++}}
$$

where $a=\frac{\sigma_{h_{++}}^{2}}{\sigma_{h_{++}}^{2}+\frac{N_{p}}{2}(1+K)}=\left[1+\frac{N_{p}(1+K)}{2\left(\left|G_{1}\right|^{2}+K\left|G_{2}\right|^{2}\right) \sigma_{h}^{2}}\right]^{-1}$. As in the previous case, $\Delta$ can be modeled as a ratio of two correlated complex Gaussian RVs.

\section{Joint channel estimation and joint equalization}

In addition to the wanted and interfering signal components on subcarrier $k, h_{++}$, and $h_{+-}$, we now also treat the corresponding quantities on the mirror subcarrier $-k$. For this, we define $h_{-+}=K_{1} h_{-} G_{2}^{*}+K_{2} h_{+}^{*} G_{1}^{*}$ and $h_{--}=K_{1} h_{-} G_{1}+K_{2} h_{+}^{*} G_{2}$. These channel coefficients can be estimated as above. All channel coefficients experience channel estimation error with variance $\sigma_{e}^{2}=$ $\frac{N_{p}}{2}\left(\left|K_{1}\right|^{2}+\left|K_{2}\right|^{2}\right)$. The biasing factor for both $h_{++}$and $h_{--}$is $a=\left[1+\frac{N_{p}(1+K)}{2\left(\left|G_{1}\right|^{2}+K\left|G_{2}\right|^{2}\right) \sigma_{h}^{2}}\right]^{-1}$, and for $h_{+-}$and $h_{-+}$it is $b=\left[1+\frac{N_{p}(1+K)}{2\left(\left|G_{2}\right|^{2}+K\left|G_{1}\right|^{2}\right) \sigma_{h}^{2}}\right]^{-1}$. The joint signal model for the mirror pair is

$$
\left[\begin{array}{l}
y_{+} \\
y_{-}^{*}
\end{array}\right]=\left[\begin{array}{l}
h_{++} \\
h_{+-}^{*} \\
h_{--}^{*}
\end{array}\right] \cdot\left[\begin{array}{l}
x_{+} \\
x_{-}^{*}
\end{array}\right]+\left[\begin{array}{l}
K_{1} n_{+}+K_{2} n_{-}^{*} \\
K_{2}^{*} n_{+}+K_{1}^{*} n_{-}^{*}
\end{array}\right]
$$

After I/Q-compensating two-tap zero forcing equalization, the symbol estimates become

$$
\hat{\mathrm{x}}=\mathrm{x}+\hat{\mathrm{H}}^{-1} \mathrm{Zx}+\hat{\mathrm{H}}^{-1} \tilde{\mathrm{n}} .
$$

The estimated data vector $\hat{\mathbf{x}}$, transmitted symbol vector $\mathbf{x}$, estimated channel matrix $\hat{\mathbf{H}}$, uncorrelated estimation error matrix $\mathbf{Z}$ and I/Q corrupted noise vector $\tilde{\mathbf{n}}$ are

$$
\begin{aligned}
& \hat{\mathrm{x}}=\left[\begin{array}{l}
\hat{x}_{+} \\
\hat{x}_{-}^{*}
\end{array}\right], \mathrm{x}=\left[\begin{array}{l}
x_{+} \\
x_{-}^{*}
\end{array}\right], \hat{\mathrm{H}}=\left[\begin{array}{l}
a \hat{h}_{++} b \hat{h}_{+-} \\
b \hat{h}_{-+}^{*} a \hat{h}_{--}^{*}
\end{array}\right] \\
& \mathbf{Z}=\left[\begin{array}{l}
z_{++} z_{+-} \\
z_{-+}^{*} z_{--}^{*}
\end{array}\right], \tilde{\mathrm{n}}=\left[\begin{array}{l}
K_{1} n_{+}+K_{2} n_{-}^{*} \\
K_{2}^{*} n_{+}+K_{1}^{*} n_{-}^{*}
\end{array}\right]
\end{aligned}
$$

From (11) and (12), the estimated symbol on subcarrier can be written as

$$
\hat{x}_{+}=x_{+}+\alpha_{+} x_{+}+\alpha_{-} x_{-}^{*}+\alpha_{0},
$$

where $\alpha_{ \pm}=\left(a \hat{h}_{--}^{*} z_{+ \pm}-b \hat{h}_{+-} z_{- \pm}^{*}\right) / D, \alpha_{0}=\left(a \hat{h}_{--}^{*} \tilde{n}_{+}-\right.$ $\left.\mathrm{b} \hat{h}_{+-} \tilde{n}_{-}^{*}\right) / D$, and the determinant of $\hat{\mathbf{H}}$ is $D=a^{2} \hat{h}_{++}$ $\hat{h}_{--}^{*}-b^{2} \hat{h}_{+-} \hat{h}_{-+} \approx a^{2} \hat{h}_{++} \hat{h}_{--}^{*}$, where the approximation 
is due to $\left|K_{2}\right|^{2}<<\left|K_{1}\right|^{2}$ and $\left|G_{2}\right|^{2}<<\left|G_{1}\right|^{2}$. Now the displacement vector can be approximated to

$$
\begin{aligned}
\Delta \approx & \frac{z_{++} x_{+}+z_{+-} x_{-}^{*}+\tilde{n}_{+}}{a \hat{h}_{++}} \\
& -\frac{b \hat{h}_{+-}}{a^{2} \hat{h}_{++} \hat{h}_{--}^{*}}\left(z_{-+}^{*} x_{+}+z_{--}^{*} x_{-}^{*}+\tilde{n}_{-}^{*}\right) .
\end{aligned}
$$

For the biasing factors, $a \geq b$ holds for all possible signalto-noise ratios (SNR) values, and $\frac{\hat{h}_{+-}}{\hat{h}_{++} \hat{h}_{--}^{*}}$ is very small. Hence, the displacement vector $\Delta$ can be approximated by neglecting the latter part of (14) to

$$
\Delta \approx \frac{z_{++} x_{+}+z_{+-} x_{-}^{*}+K_{1} n_{+}+K_{2} n_{-}^{*}}{a \hat{h}_{++}} .
$$

Now, $z_{+-}$is independent from $\hat{h}_{++}$and then the displacement vector can be modeled as a ratio of two independent complex Gaussian RVs.

\section{Probability density function of the displacement vector}

The displacement vectors of first two schemes (6) and (9) can be modeled as quotients of two correlated complex Gaussian random variables and the last scheme (15) can be treated as a quotient of two independent complex Gaussian random variables. For all cases, let us define the numerator and the denominator as $\Delta_{N}=\delta_{+} x_{+}+$ $\delta_{-} x_{-}^{*}+K_{1} n_{+}+K_{2} n_{-}^{*}$ and $\Delta_{D}=a \hat{h}_{++}$. The multiplicative factors $\delta_{+}$and $\delta_{-}$, and the biasing factors $a$ and $b$ are different for the different cases described before. Both RVs, $\Delta_{N}$ and $\Delta_{D}$ have zero mean. Their variances are $\sigma_{N}^{2}=\sigma_{\delta_{+}}^{2} P_{+}+\sigma_{\delta_{-}}^{2} P_{-}+N_{0}\left(\left|K_{1}\right|^{2}+\left|K_{2}\right|^{2}\right)$ and $\sigma_{D}^{2}=a^{2} \hat{\sigma}_{h_{++}}^{2}$, conditioned on a symbol pair $x_{+}$and $x_{-}$. The energies of symbols on the subcarrier and its mirror are given by $P_{ \pm}=\left|x_{ \pm}\right|^{2}$. The numerator and the denominator can be represented in a polar form with corresponding magnitudes by $r_{N}$ and $r_{D}$, and angles by $\theta_{N}$ and $\theta_{D}$. The joint distribution can be found in [19];

$$
\begin{aligned}
& f\left(r_{N}, r_{D}, \theta_{N}, \theta_{D}\right) \\
& \quad=\pi^{-2} \operatorname{det}(\Phi) r_{N} r_{D} e^{-\left(\varphi_{11} r_{N}^{2}+\varphi_{22} r_{D}^{2}+2 r_{N} r_{D} \cos \left(\theta_{D}-\theta_{N}+\chi_{12}\right)\right)}
\end{aligned}
$$

where $\Phi$ is the inverse covariance matrix of $\Delta=\left[\Delta_{D} \Delta_{N}\right]$ with correlation coefficient $\rho_{\Delta}$

$$
\begin{aligned}
\Phi & =\left[\begin{array}{cc}
\varphi_{11} & \varphi_{12} \\
\varphi_{12}^{*} & \varphi_{22}
\end{array}\right]=\frac{1}{\sigma_{N}^{2} \sigma_{D}^{2}-\left|\rho_{\Delta}\right|^{2}}\left[\begin{array}{cc}
\sigma_{N^{*}}^{2} & -\rho_{\Delta} \\
-\rho_{\Delta}^{*} & \sigma_{D}^{2}
\end{array}\right], \\
\varphi_{12} & =-\rho_{\Delta}=\left|\rho_{\Delta}\right| e^{j \chi_{12}}
\end{aligned}
$$

Hence, $\varphi_{11} / \varphi_{22}=\sigma_{N}^{2} / \sigma_{D}^{2}$ and correlation coefficients for first two schemes discussed above are $\rho_{\Delta}=\left|K_{1}\right|^{2} \sigma_{h}^{2}$ $\left((1+K) G_{1}^{*} G_{2}^{*}+\left|G_{2}\right|^{2}+K\left|G_{1}\right|^{2}\right)$ and $\rho_{\Delta}=\left|K_{1}\right|^{2}(1+K)$
$G_{1}^{*} G_{2}^{*} \sigma_{h}^{2}$, respectively. For the last scheme it becomes zero. Let us define the magnitude $r=r_{N} / r_{D}$ and the phase $\theta=$ $\theta_{D}-\theta_{N}$ of the displacement vector. These follow the joint distribution

$$
f_{\Delta}(r, \theta)=\frac{2}{\pi} \frac{r}{\left[\sigma_{N}^{2}+\sigma_{D}^{2} r^{2}+2 r\left|\rho_{D}\right| \cos \left(\theta+\chi_{12}\right)\right]^{2}} .
$$

For the derivation of SER, it is convenient to have a PDF of $\Delta$ in Cartesian coordinates instead of polar form. The joint PDF $f_{\Delta}(r, \theta)$ is given by $f_{\Delta}(x, y)=\frac{1}{r} f_{\Delta}(r, \theta)$ where $x=r \cos \theta$ and $y=r \sin \theta$;

$$
f_{\Delta}(x, y)=\frac{2}{\pi}\left[\frac{1}{\sigma_{N}^{2}+\sigma_{D}^{2}\left(x^{2}+y^{2}\right)+2\left|\rho_{\Delta}\right|\left(x \cos \chi_{12}-y \sin \chi_{12}\right)}\right]^{2}
$$

When the numerator and the denominator are independent, the joint distribution can be simplified to

$$
f_{\Delta}(x, y)=\frac{2}{\pi}\left[\frac{1}{\sigma_{D}^{2}\left(x^{2}+y^{2}\right)+\sigma_{N}^{2}}\right]^{2} .
$$

\section{M-QAM symbol error rate}

In this section, we derive the SER expression in an OFDM system with I/Q imbalance, imperfect channel estimation and independent fading.

\section{Symbol error rate for M-QAM symbol}

We focus on the reception of regular M-QAM signals which are most commonly used in multi-carrier systems. The constellation diagram for 16-QAM with basic constellation boundaries is shown in Figure 2. The amplitude levels of adjacent symbols in both I and Q branches are separated by the distance $d$. Assuming equi-probable symbol transmission of a rectangular $M$-QAM consisting of direct product of $M_{i}$-PAM constellation $M=M_{1} \cdot M_{2}$, the average power is $E_{s}=\left(M_{1}^{2}+M_{2}^{2}-2\right) d^{2} / 12$. When the displacement vector moves the received signal to the decision region of another constellation point, a symbol error occurs. To simplify the analysis, we determine two types of error events with corresponding probabilities. In events $A_{1}$, there is an error in the I- or Q-branch, but not in both. For a given constellation point, up to four kinds of $A_{1}$ events may occur, due to a displacement in one of the four directions $\pm \mathrm{I}, \pm \mathrm{Q}$. In events $A_{2}$, there is an error in both branches, and similarly up to four kinds of $A_{2}$ events may occur for a given constellation point. The constellation points may be split to three classes-corner, edge and middle points. We denote these with subscripts $\mathcal{C}, \mathcal{E}$, and $\mathcal{M}$, respectively. The multiplicities of the error events 


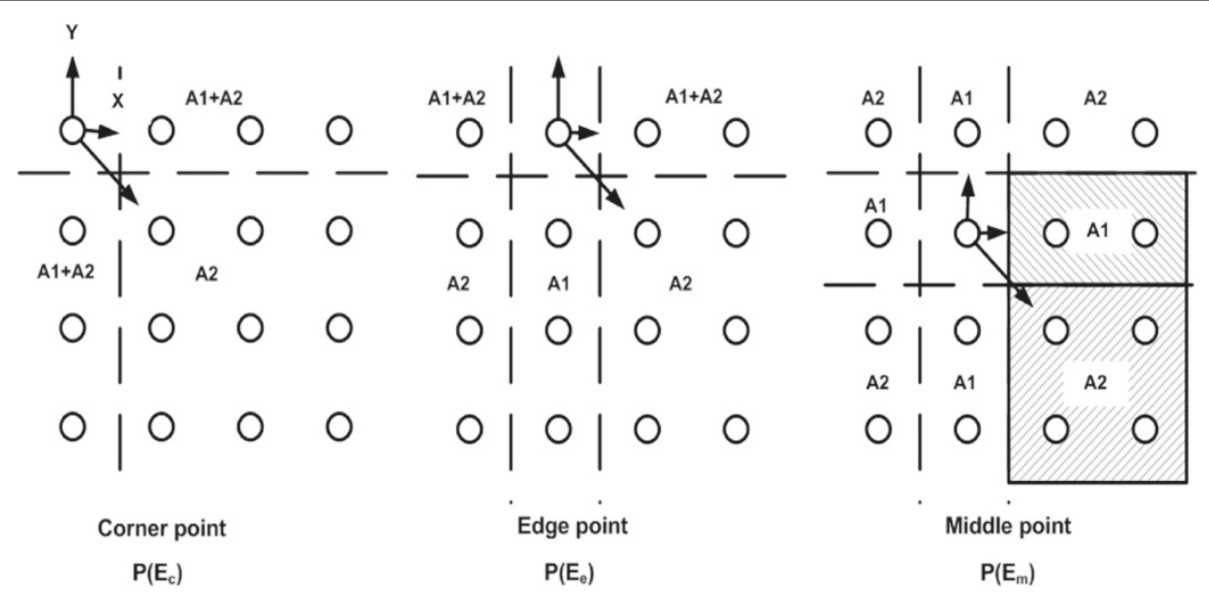

Figure 2 Distinguished areas for the occurrence of symbol errors in 16-QAM constellation. The distance between constellation points is $d$. $A_{1}$ and $A_{2}$ are the corresponding disjoint error probability areas.

of different types are different for the different classes, as shown in Figure 2. The energy of the desired symbol $P_{+}=P_{m, n}$ can be written as

$$
\begin{aligned}
\frac{P_{m, n}}{d^{2}} & =(1+2 m)^{2}+(1+2 n)^{2}, m=0, \ldots, \bar{M}_{1}-1, \\
n & =0, \ldots, \bar{M}_{2}-1
\end{aligned}
$$

where $\bar{M}_{i}=M_{i} / 2, i=1,2, m$ and $n$ are integer indices which represent the desired symbol. Similarly, the energy of the image symbol $P_{-}=P_{p, q}$ can be written by the indices $p$ and $q$ with the same range as $m$ and $n$, respectively.

Given the joint PDF in the Cartesian plane, the error probabilities $\mathrm{P}\left(\Delta \in A_{1}\right)$ and $\mathrm{P}\left(\Delta \in A_{2}\right)$ can be evaluated for a given pair of desired and mirror symbol characterized by the indices $m, n$ and $p, q$.

$$
\begin{aligned}
& \Gamma_{m, n, p, q}^{1}=\mathrm{P}\left(\Delta \in A_{1}\right)=\int_{-\infty}^{-d / 2} \int_{-d / 2}^{+d / 2} f_{\Delta}(x, y) d x d y \\
& \Gamma_{m, n, p, q}^{2}=\mathrm{P}\left(\Delta \in A_{2}\right)=\int_{-\infty}^{-d / 2} \int_{-\infty}^{-d / 2} f_{\Delta}(x, y) d x d y
\end{aligned}
$$

However, for the receiver I/Q imbalance closed form solutions are exist, for generic covariance matrix the integrals above are not solvable in closed forms (when transmitter I/Q imbalance is very small then $\rho_{\Delta} \rightarrow 0$ and hence the distribution of the displacement vector follows (20)). To progress with the analysis a first order approximation can be applied. Taking the first two terms in the Taylor series expansion around $\left|\varphi_{12}\right|=\left|\rho_{\Delta}\right|=0$ yields

$$
\begin{aligned}
f_{\Delta}(x, y) \approx & \frac{2 c^{2}}{\pi\left(c^{2}+x^{2}+y^{2}\right)^{2}} \\
& -\frac{8\left|\rho_{\Delta}\right| c^{2}\left(x \cos \chi_{12}-y \sin \chi_{12}\right)}{\pi\left(c^{2}+x^{2}+y^{2}\right)^{3}}
\end{aligned}
$$

where $c^{2}=\varphi_{11} / \varphi_{22}=\sigma_{N}^{2} / \sigma_{D}^{2}$. Now we get

$$
\begin{aligned}
\Gamma_{m, n, p, q}^{1}= & \eta_{0}-\frac{4 \eta_{0}}{\pi} \arctan \eta_{0}-\frac{8\left|\rho_{\Delta}\right| c^{2} \sin \chi_{12}}{\pi d^{3}} \\
& \times\left[\eta_{0} \eta_{1}+\frac{2}{\eta_{0}^{3}} \arctan \eta_{0}\right] \\
\Gamma_{m, n, p, q}^{1}= & \frac{1}{2}-\eta_{0}+\frac{2 \eta_{0}}{\pi} \arctan \eta_{0} \\
& -\frac{4\left|\rho_{\Delta}\right| c^{2}\left(\cos \chi_{12}-\sin \chi_{12}\right)}{\pi d^{3}} \\
& \times\left[\eta_{0} \eta_{1}+\frac{2}{\eta_{0}^{3}}\left(\pi-2 \arctan \eta_{0}\right)\right]
\end{aligned}
$$

where $\eta_{0}=\left(1+4 \lambda_{m, n, p, q}\right)^{-1 / 2}, \eta_{1}=\left(1+2 \lambda_{m, n, p, q}\right)^{-1 / 2}$ and $\lambda_{m, n, p, q}=c^{2} / d^{2}$. In these expressions,

$$
\begin{aligned}
\lambda_{m, n, p, q}= & \frac{M_{1}^{2}+M_{2}^{2}-2}{12 E_{s}} \\
& \times\left\{\frac{\sigma_{\delta_{+}}^{2} P_{m, n}+\sigma_{\delta_{-}}^{2} P_{p, q}+N_{0}\left(\left|K_{1}\right|^{2}+\left|K_{2}\right|^{2}\right)}{a^{2} \hat{\sigma}_{h}^{2}}\right\} .
\end{aligned}
$$

The error probability can be evaluated by considering the three classes of constellation points separately which is depicted in Figure 2. The conditional probabilities can be represented using disjoint events $A_{1}$ and $A_{2}$. Then 
we can derive the error probabilities for the three set of constellation points, corner, edge and middle.

$$
\mathrm{P}_{s}\left(\mathrm{E}_{\mathcal{C} / \mathcal{E} / \mathcal{M}} \mid x_{+}, x_{-}\right)=\zeta_{\mathcal{C} / \mathcal{E} / \mathcal{M}}^{1} \Gamma_{m, n, p, q}^{1}+\zeta_{\mathcal{C} / \mathcal{E} / \mathcal{M}}^{2} \Gamma_{m, n, p, q}^{2}
$$

and $\Gamma_{m, n, p, q}^{i}$ is a function of both desired and image symbols $x_{+}=[(1+2 m)+j(1+2 n)] d$ and $x_{-}=$ $[(1+2 p)+j(1+2 q)] d$. The multiplicities are

$$
\begin{array}{r}
\zeta_{\mathcal{C}}^{1}=2, \zeta_{\mathcal{E}}^{1}=3, \zeta_{\mathcal{M}}^{1}=4 \\
\zeta_{\mathcal{C}}^{2}=3, \zeta_{\mathcal{E}}^{2}=\zeta_{\mathcal{M}}^{2}=4
\end{array}
$$

The conditioning is removed by averaging over all possible desired and mirror symbol points. First removing the conditioning over the mirror symbol, we define $\Lambda_{m, n}^{i}=$ $\sum_{p=0}^{\bar{M}_{1}-1} \sum_{q=0}^{\bar{M}_{2}-1} \Gamma_{m, n, p, q}^{i}$.

As the channel coefficients on mirror carriers are independent, the average SER for the given OFDM system equals the average SER per mirror carrier pair. The contribution to the symbol error probabilities due to corner, edge and middle points now become

$$
\begin{aligned}
\mathrm{P}_{s}\left(\mathrm{E}_{\mathcal{C} / \mathcal{E} / \mathcal{M}} \mid m, n\right)= & \frac{1}{M}\left(\zeta_{\mathcal{C} / \mathcal{E} / \mathcal{M}}^{1} \Lambda_{m, n}^{1}+\zeta_{\mathcal{C} / \mathcal{E} / \mathcal{M}}^{2} \Lambda_{m, n}^{2}\right) \\
\mathrm{P}_{s}\left(\mathrm{E}_{\mathcal{C} / \mathcal{E} / \mathcal{M}}\right)= & \frac{1}{M} \sum_{m=0}^{\bar{M}_{1}-1} \sum_{n=0}^{1} \bar{M}_{2}-1 \\
& +\zeta_{\mathcal{C} / \mathcal{E} / \mathcal{E} / \mathcal{M}}^{2} \Lambda_{m, n}^{1}
\end{aligned}
$$

where $M$ is the size of the constellation. The average SER becomes

$$
\mathrm{P}_{s}(\mathrm{E})=\frac{1}{M}\left(\mathrm{P}_{s}\left(\mathrm{E}_{\mathcal{C}}\right)+\mathrm{P}_{s}\left(\mathrm{E}_{\mathcal{E}}\right)+\mathrm{P}_{s}\left(\mathrm{E}_{\mathcal{M}}\right)\right)
$$

These expressions lead to evaluate the average SER for all three cases, with the corresponding intermediate variables derived in Section 'Signal models with channel estimation errors'

\section{Closed form expression for SER}

Here, we refine the analysis for square QAM constellations with $\sqrt{M}$ even. The probability density function $f_{\Delta}(x, y)$ depends on the transmitted symbol energies on mirror subcarriers. The symbol energy distribution among the four quadrants is identical and symmetric around the diagonal points, then it is sufficient to analyze one quadrant. Thus, (24) can be further simplified to

$$
\lambda_{m, n, p, q}=\frac{M-1}{6}\left(\mu_{0} P_{m, n}+\mu_{1} P_{p, q}+\mu_{2}\right),
$$

where the pre-factors $\mu_{0}, \mu_{1}$, and $\mu_{2}$ can be found in Table 1 . SNR is denoted by $\gamma=E_{s} / N_{0}$.

The sums $\Lambda_{m, n}^{i}$ over the mirror symbols can be decomposed in to two by considering the energy of the mirror signal of the first quadrant.

$$
\begin{aligned}
\Lambda_{m, n}^{i}= & \sum_{p=0}^{\bar{M}-1} \sum_{q=0}^{\bar{M}-1} \Gamma_{m, n, p, q}^{i}=\sum_{p=q} \Gamma_{m, n, p, q}^{i} \\
& +2 \sum_{p>q} \Gamma_{m, n, p, q}^{i}, \quad i=1,2
\end{aligned}
$$

where $\bar{M}=\sqrt{M} / 2$. The have proven

Proposition 1. The symbol-error probability of an OFDM system with independently fading subcarriers, least squares channel estimation and I/Q imbalance is

$$
\mathrm{P}(\mathrm{E})=\left(\frac{4}{M}\right)^{2} \sum_{m=0}^{\bar{M}-1} \sum_{n=0}^{\bar{M}-1}\left(\zeta_{m, n}^{1} \Lambda_{m, n}^{1}+\zeta_{m, n}^{2} \Lambda_{m, n}^{2}\right)
$$

The multiplicities are $\zeta_{m, n}^{1}=4-\delta_{m, \bar{M}-1}-\delta_{n, \bar{M}-1}$ and $\zeta_{m, n}^{2}=4-\delta_{m, \bar{M}-1} \cdot \delta_{m, \bar{M}-1}$ in terms of the Kronecker $\delta$-symbol.

Example: 4-QAM: From (32), we can derive the SER

\begin{tabular}{|c|c|c|c|}
\hline & $\mu_{0}$ & $\mu_{1}$ & $\mu_{2}$ \\
\hline No IQ-compensation & $\frac{(1+K) F_{1}\left(\left|G_{1}\right|^{2}+\left|G_{2}\right|^{2}+\frac{N_{p}}{\sigma_{h}^{2}}+2 \Re\left\{G_{1} G_{2}\right\}\right)}{E_{s}\left|F_{1}+(1+K) G_{1} G_{2}\right|^{2}}-\frac{1}{E_{s}}$ & $\frac{F_{2}\left(1+\mu_{0} E_{s}\right)}{E_{s} F_{1}}$ & $\frac{(1+K)\left(1+\mu_{0} E_{s}\right)}{\gamma F_{1} \sigma_{h}^{2}}$ \\
\hline IQ comp: channel estimation & $\frac{N_{p}(1+K)}{2 F_{1} \sigma_{h}^{2} E_{s}}$ & $\frac{F_{2}\left(1+\mu_{0} E_{S}\right)}{F_{1} E_{S}}$ & $\frac{(1+K)\left(1+\mu_{0} E_{s}\right)}{\gamma F_{1} \sigma_{h}^{2}}$ \\
\hline IQ comp: chan. est. \& equalization & $\frac{N_{p}(1+K)}{2 F_{1} \sigma_{h}^{2} E_{s}}$ & $\frac{\mu_{0}\left(1+\mu_{0} E_{S}\right)}{1+\mu_{0} E_{s} \frac{F_{1}}{F_{2}}}$ & $\frac{(1+K)\left(1+\mu_{0} E_{s}\right)}{\gamma F_{1} \sigma_{h}^{2}}$ \\
\hline
\end{tabular}
for 4-QAM easily. There is only one constellation point inside the first quadrant, which is a corner point.

$$
\mathrm{P}(\mathrm{E})=2 \Lambda_{1}^{1}+3 \Lambda_{1}^{2}
$$

where $\Lambda_{1}^{1}=\Gamma_{1,1}^{1}, \Lambda_{1}^{2}=\Gamma_{1,1}^{2}$, and $\lambda_{1,1}=\frac{M-1}{6}\left\{2 \mu_{0}+\right.$ $\left.2 \mu_{1}+\mu_{2}\right\}$.

Example: 16-QAM: As shown in Figure 2, in the first quadrant there is one corner point $m=1$ with energy $P_{1}=2$, two edge points $m=2,3$ with energy $P_{2}=10$,

Table $1 \mu_{0}, \mu_{1}$, and $\mu_{2}$ with $F_{1}=\left|G_{1}\right|^{2}+K\left|G_{2}\right|^{2}$ and $F_{2}=\left|G_{2}\right|^{2}+K\left|G_{1}\right|^{2}$ for three estimation and equalization processes 
and one middle point $m=4$ with energy $P_{4}=18$. The SER expression becomes

$$
\mathrm{P}(\mathrm{E})=\frac{1}{2^{4}}[\underbrace{2 \Lambda_{1}^{1}+3 \Lambda_{1}^{2}}_{\text {corner point }}+\underbrace{2\left\{3 \Lambda_{2}^{1}+4 \Lambda_{2}^{2}\right\}}_{2 \text { edge points }}+\underbrace{4 \Lambda_{4}^{1}+4 \Lambda_{4}^{2}}_{\text {middle point }}]
$$

where $\Lambda_{m}^{i}=\Gamma_{m, 1}^{i}+2 \Gamma_{m, 2}^{i}+\Gamma_{m, 4}^{i}, i=1,2$.

\section{Bit error rate for M-QAM symbol transmission}

Next, we calculate the bit error rate of an I/Q-distorted system, assuming square QAM symbols, with even $\sqrt{M}$, and Gray-coded bits. For Gray-coded M-QAM, the decision boundaries for horizontal signals are independent of the vertical signal levels, and vice versa [20]. Thus for perfect channel information it is sufficient to calculate the BER for horizontal $\sqrt{M}$-PAM. The probability distribution of the displacement vector in (19) is not circularly symmetric over given set of symbol pair $x_{+}$and $x_{-}$thus the average error probability along the horizontal and vertical directions are not exactly the same. Therefore, both horizontal and vertical PAM error probabilities need to be evaluated. The individual error probabilities along both directions depend on the marginal densities. Next, the error probability on horizontal vertical PAM can be considered.

Consider a M-QAM symbol $x_{+}=x_{r+}+j x_{i+}$, with distance $d$ between adjacent symbols. Let $\bar{\Delta}=\Re(\Delta)$ be the real part of the displacement error vector corresponding to the error probabilities along the real axis. For horizontal PAM, the BER for a given imaginary part of a symbol $x_{i+}$ and the decision boundary $B^{k}$ can be defined as the probability that $\bar{\Delta}$ and $x_{r+}$ fall on the different sides of the decision boundary. Thus the conditional BER for the horizontal part for a particular bit position $b_{k}$ of M-QAM for a given imaginary part of the desired symbol $x_{i+}$ and the image signal $x_{-}$is

$$
\tilde{\mathrm{P}}_{H}\left(b_{k} \mid x_{i+}, x_{-}\right)=\left\{\begin{array}{l}
\tilde{\mathrm{P}}\left(\bar{\Delta}<B^{k} \mid x_{i+}, x_{-}\right), x_{r+}>B^{k} \\
\tilde{\mathrm{P}}\left(\bar{\Delta}>B^{k} \mid x_{i+}, x_{-}\right), x_{r+}<B^{k}
\end{array}\right.
$$

The conditioning on the imaginary part $x_{i+}$ and the mirror symbol $x_{-}$is removed by averaging over all possibilities Hence, the total error probability of horizontal PAM becomes

$$
\mathrm{P}_{\mathrm{H}}=\frac{2}{M^{2} \log _{2} \sqrt{M}} \sum_{\forall x_{i+}} \sum_{\forall x_{-}} \sum_{k=1}^{\log _{2} \sqrt{M}} \tilde{\mathrm{P}}_{\mathrm{H}}\left(b_{k} \mid x_{i+}, x_{-}\right)
$$

Here $\forall x_{ \pm}$represents the all QAM constellation points which transmit on either subcarrier or its mirror. Similarly, the bit error rate of vertical PAM can be derived. For square QAM these BERs are the same.

The inner sum of the equations above represents the sum of individual bit error probabilities. For a given $\sqrt{M}$ PAM signal with $\sqrt{M}$ even, there are an equal number of points, $\bar{M}=\sqrt{M} / 2$, on the positive and negative side of the constellation, and the allocated number of bits per constellation point is $\bar{L}=\log _{2} \sqrt{M}$. The decision boundaries can be parameterized as $B_{ \pm r}^{k}= \pm 2^{\bar{L}-k}(2 r-1) d, k>$ 1 , with a boundary index $r$ and a bit index $k$, c.f., Figure 3 .

The decision boundaries on right side of $B_{1}^{1}$ have positive boundary index, $B_{r}^{k}$, and boundaries on the left side have negative indices, $B_{-r}^{k}$. The number of boundaries per side corresponding to the $k^{t h}$ bit position is $R=2^{k-2}, k>$ 1 , where $r=1, \ldots, R$. The sum over the BERs of the $\sqrt{M}$ bits becomes

$$
\begin{aligned}
& \mathrm{P}_{\Sigma}\left(\mathrm{E} \mid x_{i+}, x_{-}\right)=\sum_{k=1}^{\bar{L}} \tilde{\mathrm{P}}_{\mathrm{H}}\left(b_{k} \mid x_{i+}, x_{-}\right) \\
& =\sum_{m=1}^{\bar{M}} \mathrm{P}\left(\bar{\Delta}_{m}>\frac{2 m-1}{2} d\right)+\sum_{k=2} \sum_{m=1}^{\bar{M}} \sum_{r=1}^{R} \\
& \quad \times\left[s_{r}^{m} \mathrm{P}\left(\bar{\Delta}_{m}>\left|\frac{\bar{M}(2 r-1)-R(2 m-1)}{2 R}\right| d\right)\right. \\
& \left.\quad+s_{-r}^{m} \mathrm{P}\left(\bar{\Delta}_{m}>\frac{\bar{M}(2 r-1)+R(2 m-1)}{2 R} d\right)\right]
\end{aligned}
$$

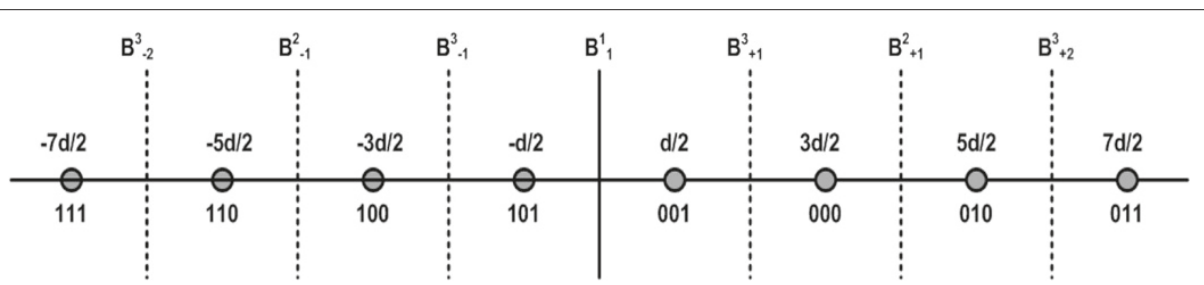

Figure 3 Gray coded 8-PAM constellation with decision boundaries $B_{ \pm r}^{k}$, where $k$ and $r$ represent the corresponding bit index and boundary index. 
The coefficients $s_{ \pm r}^{m}$, depending on the bit position index $m$ and the boundary index $r$ are

$$
\begin{aligned}
& s_{r}^{m}=\prod_{\tilde{r}=1, \tilde{r} \neq r}^{R} \operatorname{sgn}\left(\frac{1}{2}+\frac{\bar{M}(2 \tilde{r}-1)}{R}-m\right)(-1)^{r+1} \\
& s_{-r}^{m}=\prod_{\tilde{r}=1}^{R} \operatorname{sgn}\left(\frac{1}{2}+\frac{\bar{M}(2 \tilde{r}+1)}{R}-m\right)(-1)^{r+1}
\end{aligned}
$$

Note that for $k=2$, the coefficients $s_{ \pm r}^{m}=1, \forall m$. For the above square QAM system with unequal error probabilities on horizontal and vertical PAM, the average BER thus simplifies to [20]

$$
\mathrm{P}_{\mathrm{M}}=\frac{1}{2}\left(\mathrm{P}_{H}+\mathrm{P}_{V}\right)
$$

The evaluation of the individual probabilities $\mathrm{P}\left(\bar{\Delta}_{m}>\right.$ $\kappa d)$ is not as simple as in the Gaussian case, because each desired and mirror symbol combination, contributes their own PDF which is centered around the corresponding bit position. Evaluating these, we have

Proposition 2. The bit-error probability of an OFDM system with independently fading subcarriers, least squares channel estimation and I/Q imbalance is

$$
\begin{aligned}
\mathrm{P}_{\mathrm{H}}= & a_{0} \sum_{n=1}^{\bar{M}} \sum_{p=1}^{\bar{M}} \sum_{q=1}^{\bar{M}}\left\{\sum_{m=1}^{\bar{M}} f\left(\lambda_{m, n, p, q}, \kappa_{1}(m)\right)\right. \\
& +\sum_{k=2}^{\bar{L}} \sum_{m=1}^{\bar{M}} \sum_{r=1}^{R}\left[s_{r}^{m} f\left(\lambda_{m, n, p, q}, \kappa_{2}(m, r)\right)\right. \\
& \left.\left.+s_{-r}^{m} f\left(\lambda_{m, n, p, q}, \kappa_{3}(m, r)\right)\right]\right\}
\end{aligned}
$$

where $\mathrm{P}\left(\bar{\Delta}_{m}>\kappa_{i} d\right)=f\left(\lambda_{m, n, p, q}, \kappa_{i}\right)$ with three different $\kappa$ values: $\kappa_{1}=(2 m-1) d / 2$, $\kappa_{2}=|(\bar{M}(2 r-1)-R(2 m-1)) / 2 R \quad| \quad d$ and $\kappa_{3}=(\bar{M}(2 r-1)+R(2 m-1)) d / 2 R$. Furthermore, $a_{0}=2^{4-2 \bar{L}} / \bar{L}, \bar{M}=\sqrt{M} / 2, \bar{L}=\log _{2} \sqrt{M}$ and $R=2^{k-2}$. The coefficients $s_{ \pm r}^{m}$ can be found in (37). The I/Qimbalance affects the terms $\lambda_{m, n, p, q}$ of Equation (24). Similarly, for the vertical PAM individual error probabilities given by $\mathrm{P}\left(\bar{\Delta}_{n}>\kappa_{i} d\right)=g\left(\lambda_{m, n, p, q}, \kappa_{i}\right)$ which can be derived from its own marginal distribution $f_{\Delta}(y)$.

Proof. The marginal distributions of $\Delta$ is evaluated first from (19),

$$
\begin{aligned}
& f_{\Delta}(x)=\frac{c^{2}-b^{2}}{\left(x^{2}+2\left|\rho_{\Delta}\right| \cos \chi_{12} x-\left|\rho_{\Delta}\right|^{2} \sin ^{2} \chi_{12}+c^{2}\right)^{3 / 2}} \\
& f_{\Delta}(y)=\frac{c^{2}-b^{2}}{\left(y^{2}-2\left|\rho_{\Delta}\right| \sin \chi_{12} y-\left|\rho_{\Delta}\right|^{2} \cos ^{2} \chi_{12}+c^{2}\right)^{3 / 2}}
\end{aligned}
$$

so that

$$
\begin{aligned}
& f\left(\lambda_{m, n, p, q}, \kappa_{i}\right)=1-\frac{\kappa_{i}+\frac{\left|\rho_{\Delta}\right| \cos \chi_{12}}{d}}{\sqrt{\lambda_{m, n, p, q}+\kappa_{i}^{2}+\left(\frac{\left|\rho_{\Delta}\right| \cos \chi_{12}}{d}+\kappa_{i}\right)^{2}-\left(\kappa_{i}^{2}+\frac{\left|\rho_{\Delta}\right|^{2}}{d^{2}}\right)}} \\
& g\left(\lambda_{m, n, p, q}, \kappa_{i}\right)=1-\frac{\kappa_{i}-\frac{\left|\rho_{\Delta}\right| \sin \chi_{12}}{d}}{\sqrt{\lambda_{m, n, p, q}+\kappa_{i}^{2}+\left(\kappa_{i}-\frac{\left|\rho_{\Delta}\right| \sin \chi_{12}}{d}\right)^{2}-\left(\kappa_{i}^{2}+\frac{\left|\rho_{\Delta}\right|^{2}}{d^{2}}\right)}}
\end{aligned}
$$

where $c^{2}=\sigma_{D}^{2} / \sigma_{D}^{2}, b^{2}=\left|\rho_{\Delta}\right|^{2} / \sigma_{D}^{2}$. The corresponding $\kappa_{i}$ values for vertical PAM can be obtained by replacing $m$ with $n$. Then, the average BER can be evaluated from (38)

\section{Analytical limits}

From the derived results, known results for error rates in AWGN and Rayleigh channels with and without I/Q imbalance in the absence of channel estimation errors can be derived for square M-QAM constellation.

\section{AWGN channel without I/Q imbalance}

As a reference case, in AWGN with perfect receivers $\left(K_{1}=1, K_{2}=0 h(k)=1\right),(24)$ can be reduced to

$$
\begin{aligned}
& \Gamma^{1}=Q\left(\sqrt{\frac{3 \gamma}{M-1}}\right)-2 Q^{2}\left(\sqrt{\frac{3 \gamma}{M-1}}\right) \\
& \Gamma^{2}=Q^{2}\left(\sqrt{\frac{3 \gamma}{M-1}}\right)
\end{aligned}
$$

where $Q(\cdot)$ is a Gaussian Q-function, and $\gamma$ is the SNR defined as before. Hence we recover the well known expression [21]

$$
\begin{aligned}
\mathrm{P}(E) & =4 \Gamma_{1} \frac{M-\sqrt{M}}{M}+4 \Gamma_{2} \frac{M-1}{M} \\
& =1-\left[1-\frac{\sqrt{M}-1}{\sqrt{M}} \cdot 2 Q\left(\sqrt{\frac{3 \gamma}{M-1}}\right)\right]^{2} .
\end{aligned}
$$

\section{AWGN channel with receiver $\mathrm{I} / \mathrm{Q}$ imbalance}

In an AWGN channel with receiver I/Q imbalance, the displacement vector $\Delta$ can be represented as a complex Gaussian random variable with mean $\mu_{\Delta}=\frac{K_{2}}{K_{1}} x_{-}^{*}$ and variance $\sigma_{\Delta}^{2}=(1+K) N_{0}$. Hence the PDF of $\Delta$ becomes a complex Gaussian distribution.

$$
f(x, y)=\frac{1}{2 \pi \sigma_{\Delta}^{2}} \exp \left(-\frac{\left(x-\mu_{x}\right)^{2}+\left(y-\mu_{y}\right)^{2}}{2 \sigma_{\Delta}^{2}}\right)
$$

where $\mu_{x}=\mathfrak{R}\left\{\mu_{\Delta}\right\}$ and $\mu_{y}=\Im\left\{\mu_{\Delta}\right\}$. The SER is connected to the cumulative distribution functions (CDFs) $F_{X}(d)$ and $F_{Y}(d)$ by

$$
\mathrm{P}(E)=1-\left[1-\frac{\sqrt{M}-1}{\sqrt{M}} F_{X}(d)\right]\left[1-\frac{\sqrt{M}-1}{\sqrt{M}} F_{Y}(d)\right]
$$


where individual CDFs can be obtained by substituting corresponding means $\mu_{x}$ and $\mu_{y}$ to

$$
F(d)=Q\left(\frac{d-\mu}{\sigma_{\Delta}}\right)+Q\left(\frac{d+\mu}{\sigma_{\Delta}}\right) .
$$

The argument inside the $\mathrm{Q}$ functions can be written as $\frac{d \pm \mu}{\sigma_{\Delta}}=\sqrt{\frac{6 \gamma}{(M-1)(1+K)}}\left(1 \pm \Re / \Im\left\{\frac{\mu_{\Delta}}{d}\right\}\right)$ where $\frac{\mu_{\Delta}}{d}=$ $\sqrt{\frac{M-1}{6 E_{s}}} K x_{-}^{*}$. This result coincides with [22].

\section{Rayleigh faded AWGN channel without I/Q imbalance}

In Rayleigh fading with unit variance, Equation (24) becomes $\lambda=(M-1) / 6 \gamma$. There are four corner points, $4(\sqrt{M}-2)$ edge points and $(\sqrt{M}-2)^{2}$ middle points, respectively. Hence, Equation (29) can be reduced to

$$
\begin{aligned}
\mathrm{P}(E)= & 4 \Gamma^{1} \frac{M-\sqrt{M}}{M}+4 \Gamma^{2} \frac{M-1}{M} \\
= & 2 \frac{\sqrt{M}-1}{\sqrt{M}}(1-\hat{\gamma})-\left(\frac{\sqrt{M}-1}{\sqrt{M}}\right)^{2} \\
& \times\left(1-\hat{\gamma} \frac{4}{\pi} \tan ^{-1} \frac{1}{\hat{\gamma}}\right)
\end{aligned}
$$

where $\hat{\gamma}=\sqrt{\frac{\frac{3}{2} \gamma}{M-1+\frac{3}{2} \gamma}}$. The results coincides with the well-known error probability for Rayleigh channels [22].

Rayleigh faded AWGN channel with receiver I/Q imbalance Similarly, for an I/Q corrupted Rayleigh faded system (24) becomes

$$
\lambda=\frac{M-1}{6}\left(\frac{K\left|x_{-}\right|^{2}}{E_{s}}+\frac{1+K}{\gamma}\right)
$$

With I/Q imbalance at the receiver, Equation (29) can be reduced to

$$
\mathrm{P}(E)=\frac{M-1}{M}-\frac{\sqrt{M}-1}{M} \frac{2}{\hat{\gamma}}-\frac{(\sqrt{M}-1)^{2}}{M} \frac{4}{\pi \hat{\gamma}} \tan ^{-1} \frac{1}{\hat{\gamma}}
$$

where $\hat{\gamma}=\frac{1}{\sqrt{1+\frac{2(M-1)}{3}\left(\frac{K|x-|^{2}}{E_{S}}+\frac{1+K}{\gamma}\right)}}$, reproducing the result of [23].

\section{Simulation results}

In this section, we compare the above analytical SER/BER expressions to simulation results for an OFDM system in frequency selective fading, with transmitter and receiver $\mathrm{I} / \mathrm{Q}$ imbalance and channel estimation errors. The OFDM system discussed here is assumed properly synchronize in time and frequency, and the cyclic prefix is sufficient to remove the multipath effect completely. For the simulation, we consider different M-QAM modulations $(M=$
$16,64,256)$. The effect of $I / Q$ imbalance is investigated for different IRR values ranging from $25-55 \mathrm{~dB}$. For the worst case, we select $g=0.9$ and $\phi=2^{\circ}$ for both transmitter and receiver. The channel coefficients on mirror carrier pairs can be modeled by an independent complex Gaussian process $\mathcal{C N}(0,1)$. The noise is assumed to be white complex Gaussian $\mathcal{C N}\left(0, N_{0}\right)$ as well. Pilots on mirror carrier pairs are selected from BPSK constellation as in $[8,10]$ for channel estimation. When receiving each information symbol transmitted, a least square channel estimate is used which has been estimated from a pilot symbol transmitted with exactly the same channel. When joint channel estimation is performed, the pilots are chosen from a Walsh-Hadamard code with BPSK symbols, as in (7).

Figure 4 shows the theoretical and simulation results for the three cases of channel estimation and equalization discussed in Section 'Signal models with channel estimation errors'. When there is no IQ-compensation at the receiver (case a), SER performance is poor. The channel estimation error in (4) contains both the I/Q corrupted mirror carrier interference and the channel noise. There is an irreducible error floor visible, due to the mirror carrier interference which dominates at high SNR. When there is IQ-compensation in the channel estimation (case b), the performance is slightly better. However, the effect of I/Q imbalance in the channel noise term, not compensated in equalization, leads to an error floor as in as the previous case. In case $c$, we compensate for I/Q imbalance both in channel estimation and equalization phases. Hence the performance is better than in the other two cases, and the error floor is removed. The approximation works perfectly and matches the simulation result well. In the two first cases, the closed form analytical result matches simulations perfectly.

Figure 5 shows the behavior of the biasing factors $a$ and $b$ for the above three channel estimation and equalization techniques for a worst I/Q condition (IRR $=25 \mathrm{~dB})$ and a close-to-ideal ( $\mathrm{IRR}=55 \mathrm{~dB})$. The biasing factor on subcarrier channel estimation can be approximated to $a \approx$ $\left[1+\frac{N_{p}}{2 \sigma_{h}^{2}}\right]^{-1}$ (for the separate channel estimation and equalization $a \approx\left[1+\frac{N_{p}}{\sigma_{h}^{2}}\right]^{-1}$ ) for the above IRR range. Hence, the effect of IRR is insignificant and the AWGN term dominates the performance. Therefore, at high SNR values $a$ converges to unity Similarly, the biasing factor on mirror carrier channel estimation can be written as $b \approx[1+$ $\left.\frac{N_{p}}{2 K\left|G_{1}\right|^{2} \sigma_{h}^{2}}\right]^{-1}$. For the given channel statistics, the behavior of $b$ for a I/Q corrupted receivers dominates by the SNR. Hence, it converges to unity when SNR increases. But for a ideal receiver with $K=0$ it converges to zero irrespective of SNR and the transmitter I/Q imbalance $\left(\left|G_{1}\right|^{2} \approx 1\right)$.

The error rate performance simulations (Figures 6, 7, 8 , and 9) focus on joint channel estimation and separate 


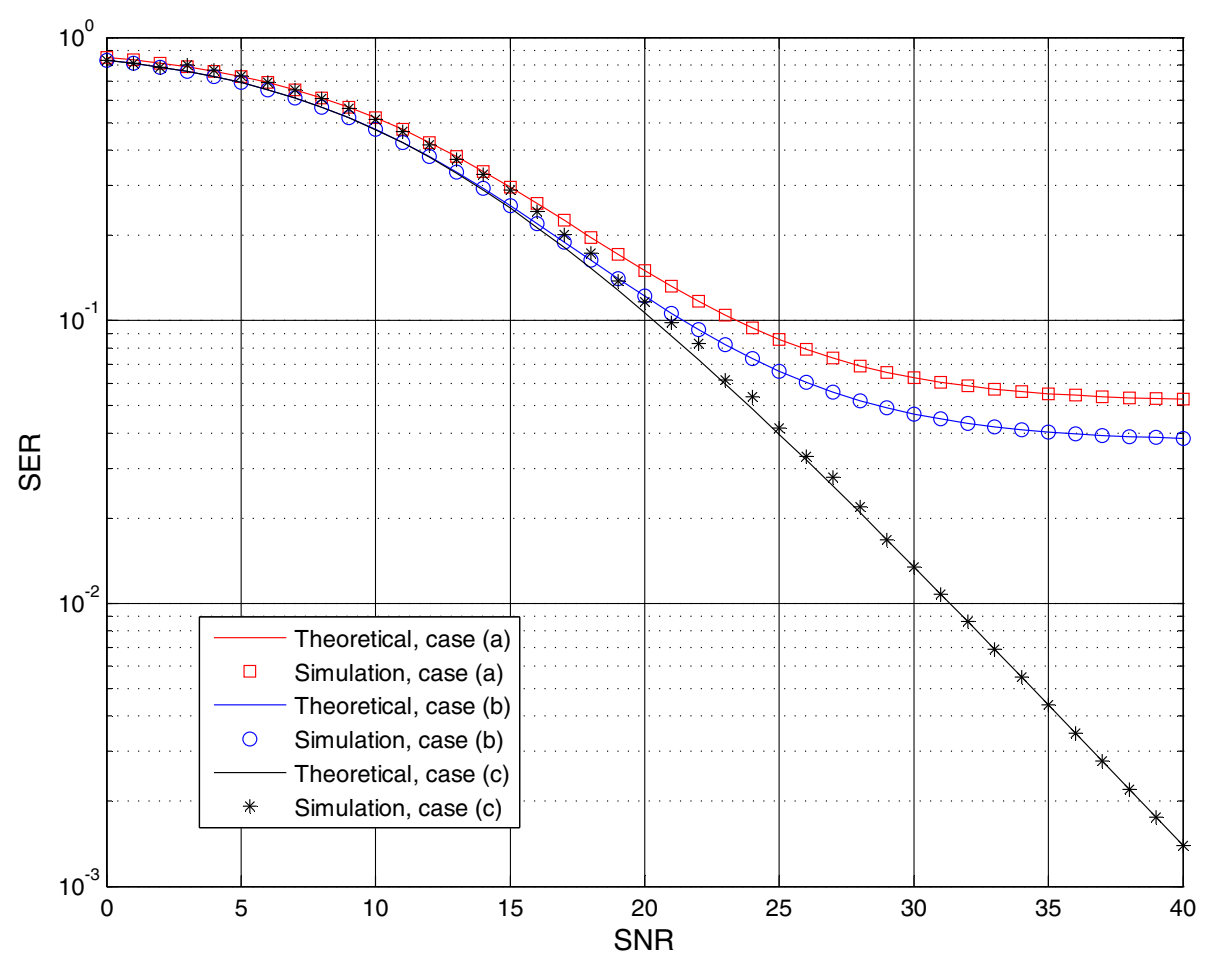

Figure 4 SER performance for the three estimation and equalization schemes. (a) Separate channel estimation with separate equalization. (b) Joint channel estimation with separate equalization. (c) Joint channel estimation with joint equalization. Simulation and theoretical results for 16-QAM with gain $g=0.9$ and phase $\phi=2^{\circ}$ for both transmitter and receiver.

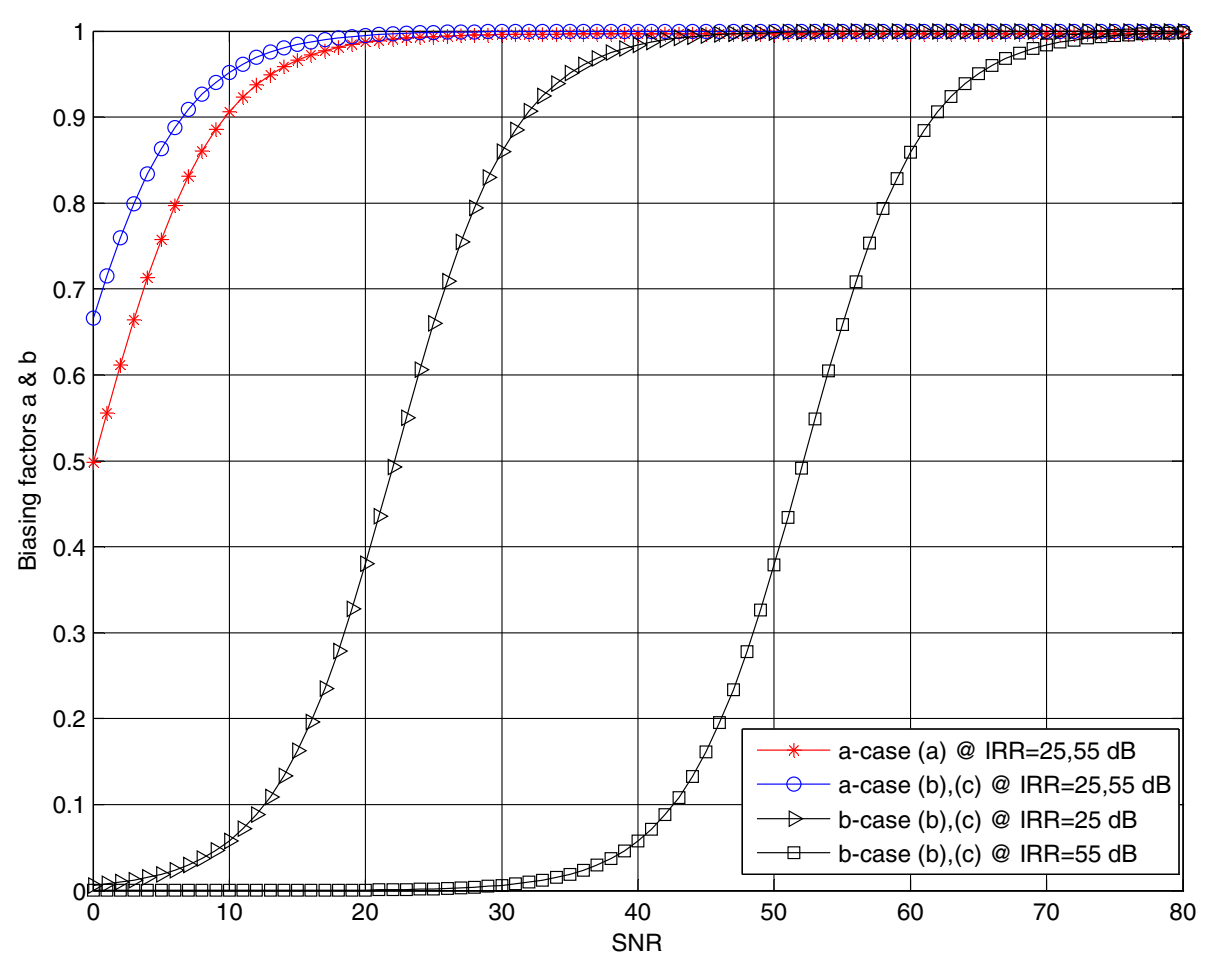

Figure 5 Behavior of the biasing factors $a$ and $b$ of the three estimation and equalization schemes for worst case (IRR $=25 \mathrm{~dB} g=0.9$ and $\phi=2^{\circ}$ ) and close-to-ideal (IRR $=55 \mathrm{~dB} g=1$ and $\phi=0.2^{\circ}$ ) transceivers. 


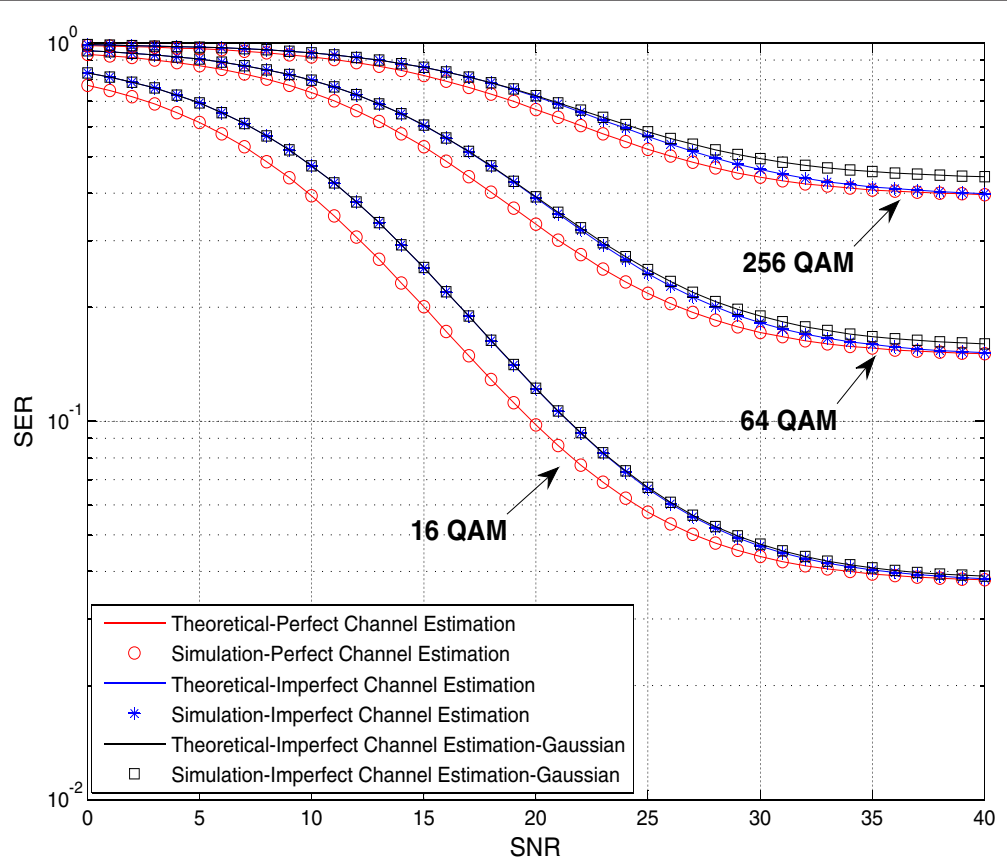

Figure 6 SER for M-QAM modulations $(M=16,64,256)$ of ZF equalization with perfect channel knowledge, imperfect channel estimation and with Gaussian approximated mirror carrier interference at IRR $25 \mathrm{~dB}$ for both transmitter and receiver.

equalization which is discussed in Section 'Signal models with channel estimation errors'.

Figure 6 depicts the SER performance of the ZF equalization on the perfect channel knowledge and the effect of imperfect channel estimation. The effect of channel estimation is simulated with Gaussian approximated mirror carrier interference as well. From Figure 4, it is clear that the biasing factor $a$ does not have any significant impact on system performance. For the relatively small $K$, $a \rightarrow a_{\text {ideal }}=\left[1+\frac{N_{p}}{\sigma_{h}^{2}}\right]^{-1}$ where $a_{\text {ideal }}$ is the biasing factor of

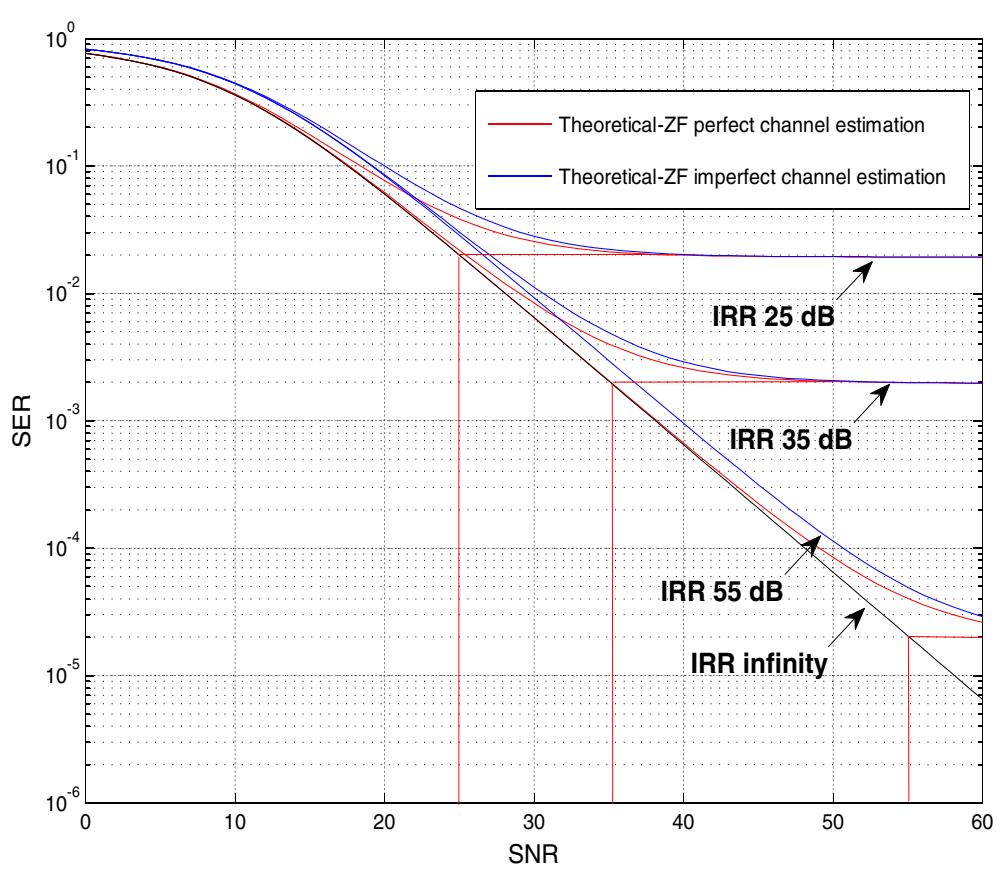

Figure 7 SER of 16-QAM modulation of ZF equalization with perfect channel estimation and with imperfect channel estimation in Rayleigh fading environment for different receiver IRR values (IRR $=\mathbf{2 5}, \mathbf{3 5}, \mathbf{5 5}, \infty \mathbf{d B}$ ). Asymptotic SINR values are marked in red. 


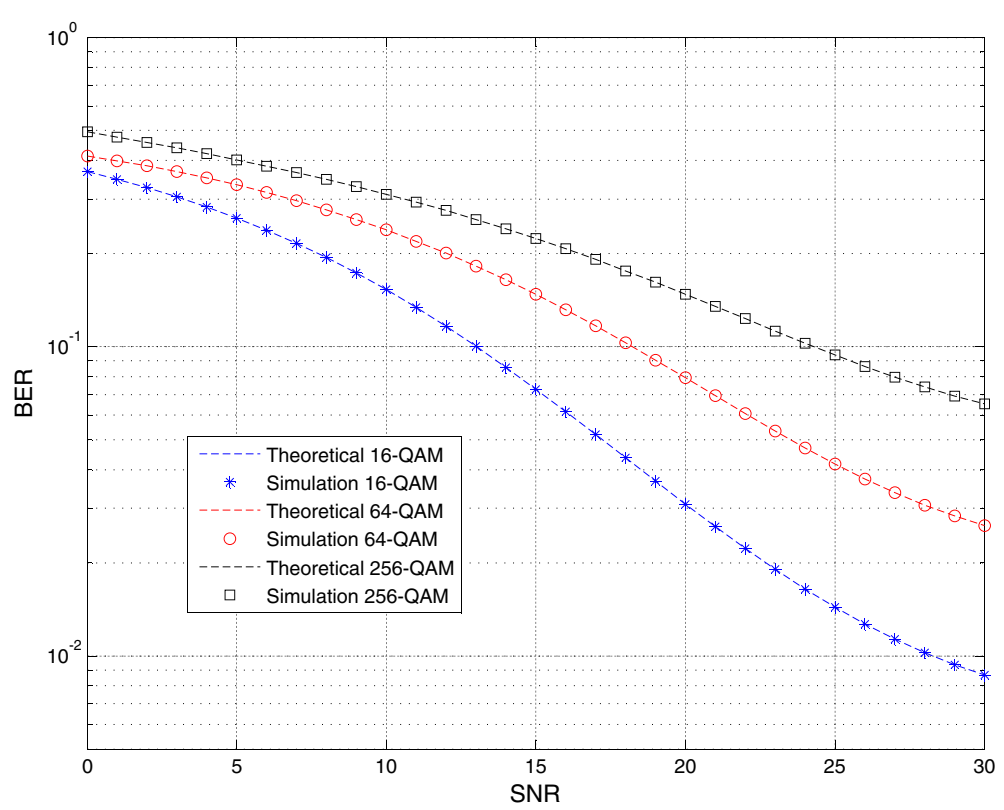

Figure 8 BER for M-QAM modulation $(M=16,64,256)$ of $Z F$ equalization against imperfect channel estimation in Rayleigh fading environment for receiver IRR $25 \mathrm{~dB}$.

an ideal receiver which again emphasis the above facts. At higher SNR, the estimation error term $z_{++}\left[\sigma_{z_{++}}^{2}=(1-\right.$ a) $\sigma_{h_{+}}^{2}$ ] converges to zero, while the biasing factor goes to unity. Therefore, the imperfect channel estimation follows the equalization with perfect channel knowledge at higher SNR values. Performance of the Gaussian approximated system shows slightly worst error floors with higher order modulations. However, it is reasonable to assume the mirror carrier interference as Gaussian distributed specially at low order modulation schemes.

The effect of transmitter I/Q imbalance is mild compared to the receiver $\mathrm{I} / \mathrm{Q}$ imbalance and rest of the simulations consider only the receiver I/Q imbalance.

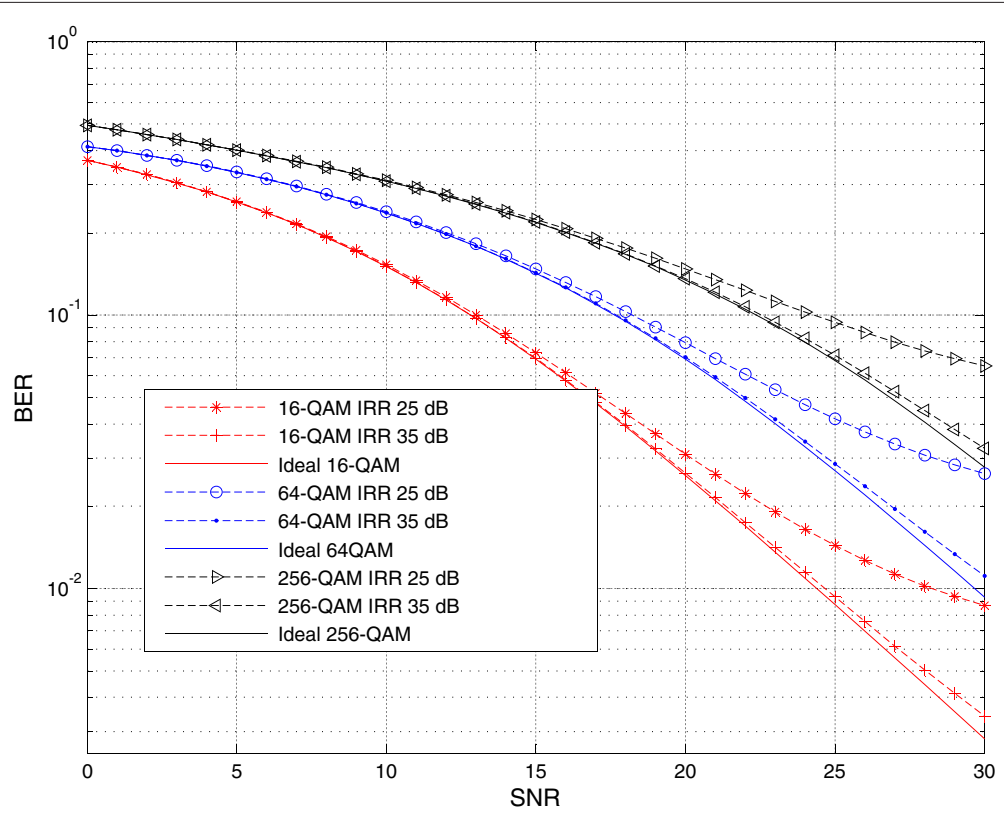

Figure 9 BER of 16, 64, 256-QAM modulation of the ZF equalization of imperfect channel estimation in Rayleigh fading environment for different receiver IRR values (IRR $=25,35, \infty$ (ideal) $\mathrm{dB}$ ). 
Figure 7 presents how the ZF equalization with channel estimation works for three different IRR values $(25$, 35 , and $55 \mathrm{~dB}$ ) with. There is an irreducible error floor because of the $\mathrm{I} / \mathrm{Q}$ induced mirror carrier interference which dominates the effective interference at high SNR region. The convergence limit changes according to the IRR value appears at the receiver. Hence, asymptotic SINR approximately equivalents to the IRR and will affects to the SER convergence as depicted in the same plot (projection on to the ideal receiver implies that the Gaussian approximation of I/Q interference). Eventually, the receiver close to ideal receiver perform better.

Then Figure 8 shows the BER performance for 16/64/256-QAM symbols. It validates the expressions derived in (39) with the simulation result for M-QAM symbols with Gray coded bit mapping. Figure 9 interprets the BER performance for 16/64-QAM with IRR $=25,35$. For comparison ideal receiver (without the I/Q imbalance $K \rightarrow 0$ ) also plotted in the same figure. For higher order modulations with higher image rejection follow the ideal receiver for a lower SNR region. But for lower modulation schemes, performance deviates more from the ideal case. But in general, system converges to a particular BER value at high SNR as a result of uncompensated mirror carrier interference.

\section{Conclusion}

Closed form expressions for SER/BER performance for the M-QAM modulated OFDM system in frequency selective Rayleigh fading channel under the effect of transmitter and receiver I/Q imbalance and imperfect channel estimation has been derived. Commonly used three channel estimation and equalization schemes are considered and performance is evaluated. If a Gaussian approximation is applied, good results for lower modulations orders are found. For higher order modulation, a Gaussian approximation slightly overestimates error floors. Channel coefficients on mirror subcarriers can be assumed uncorrelated for mirror carrier pairs which are separated more than the coherence bandwidth. In broadband communication this would hold for most of the mirror carrier pairs. The SER/BER expressions obtained here can thus be considered as upper bounds for SER/BER in finite delay spread channels, becoming tight in the limit when the bandwidth becomes large compared to the coherence bandwidth.

\section{Competing interest}

The authors declare that they have no competing interests.

Received: 27 January 2012 Accepted: 25 July 2012

Published: 25 September 2012

\section{References}

1. REPORT ITU-R M.2134, Requirements related to technical performance for IMT-Advanced radio interface(s) $\mathbf{2 1 3 4}, 8$ (2008)

2. AA Abidi, Direct-conversion radio transceivers for digital communications. IEEE J. Solid-State Circ. 30(12), 1399-1410 (1995)

3. M Windisch, G Fettweis, in Circuits and Systems ISCAS On the impact of I/Q imbalance in multi-carrier systems for different channel scenarios. (New Orleans, USA, 2007), pp. 33-36

4. M Windisch, G Fettweis, in IEEE 60th Vehicular Technology Conference, VTC2004-Fall, vol. 3 Adaptive I/Q imbalance compensation in low-IF transmitter architectures. (Los Angeles, CA, 2004), pp. 2096-2100

5. M Windisch, G Fettweis, in IEEE/SP 14th Workshop Statistical Signal Processing, SSP'07 A blind estimation and compensation of I/Q imbalance in OFDM receivers with enhancements through Kalman filtering. (Madison, Wisconsin, 2007), pp. 754-759

6. M Valkama, M Renfors, V Koivunen, Advanced methods for I/Q imbalance compensation in communication receivers. IEEE Trans. Signal Process. 49(10), 2335-2344 (2001)

7. A Tarighat, R Bagheri, AH Sayed, Compensation schemes and performance analysis of IQ imbalances in OFDM receivers. IEEE Trans. Signal Process. 53(8), 3257-3268 (2005)

8. Y Egashira, Y Tanabe, K Sato, in Vehicular Technology Conference A novel IQ imbalance compensation method with pilot-signals for OFDM system. (Montreal, CA, 2008), pp. 1-5

9. J Tubbax, A Fort, L Van der Perre, S Donnay, M Engels, M Moonen, H De Man, in GLOBECOM 2003 Joint compensation of IQ imbalance and frequency offset in OFDM systems. (San Francisco, USA, 2003), pp. 2365-2369

10. TCM Schenk, PFM Smulders, ER Fledderus, Estimation and compensation of Tx and Rx IQ imbalance in OFDM-based MIMO systems. IEEE Trans. Signal Process. 49(10), 2335-2344 (2001) (Dresden, Germany)

11. L Anttila, M Valkama, M Renfors, in Vehicular Technology Conference Efficient mitigation of frequency-selective I/Q imbalance in OFDM receivers. (Marina bay, Singapore, 2008), pp. 1-5

12. M Windisch, G Fettweis, in Proc. 9th Intl. OFDM Workshop (InOWo) Standard-independent I/Q imbalance compensation in OFDM direct-conversion receivers. (Dresden, Germany, 2004), pp. 57-61

13. M Krondorf, G Fettweis, OFDM link performance analysis under various receiver impairments. EURASIP J. Wirel. Commun. Network. 145279(11) (2008)

14. M Krondorf, G Fettweis, Numerical performance evaluation for Alamouti space time coded OFDM under receiver impairments. IEEE Trans. Wirel. Commun. 8(3), 1446-1455 (2009)

15. B Razavi, RF Microelectronics (Prentice-Hall, Upper Saddle River, 1998)

16. X Dong, $L$ Xiao, Symbol error probability of two-dimensional signalling in Ricean fading with imperfect channel estimation. IEEE Trans. Veh. Technol. 54(2), 538-549 (2005)

17. L Cao, NC Beaulieu, Exact error-rate analysis of diversity 16-QAM with channel estimation error. IEEE Trans. Commun. 52(6), 1019-1029 (2004)

18. U Oruthota, O Tirkkonen, in IWCMC Effect of imperfect channel estimation in IQ imbalanced OFDM system. (Caen, France, 2010), pp. 596-600

19. KS Miller, Complex Gaussian processes. SIAM Rev. 11(4), 544-567 (1969)

20. $Y M a, J$ Jin, Effect of channel estimation errors on M-QAM with MRC and EGC in Nakagami fading channels. IEEE Trans. Veh. Technol. 56(3), 1239-1250 (2007)

21. JG Proakis, Digital Communications, 3nd edn. (McGraw-Hill, Inc., New York, 1995)

22. MK Simon, M-S Alouini, Digital Communication Over Fading Channels, 2nd edn. (Wiley, New York, 2005)

23. M Windisch, G Fettweis, in ICC-2006 Performance degradation due to I/Q imbalance in multi-carrier direct conversion receivers: a theoretical analysis. (Istanbul, 2006), pp. 257-262

doi:10.1186/1687-1499-2012-303

Cite this article as: Oruthota and Tirkkonen: SER/BER expression for MQAM OFDM systems with imperfect channel estimation and I/Q imbalance. EURASIP Journal on Wireless Communications and Networking 2012 2012:303. 